\title{
Simultaneous Multiparametric PET/MRI with Silicon Photomultiplier PET and Ultra-High-Field MRI for Small-Animal Imaging
}

\author{
Guen Bae Ko ${ }^{1,2}$, Hyun Suk Yoon ${ }^{1,2}$, Kyeong Yun Kim ${ }^{1,2}$, Min Sun Lee ${ }^{1,3}$, Bo Yeun Yang ${ }^{1,4}$, Jae Min Jeong ${ }^{1-4}$, \\ Dong Soo Lee ${ }^{1,3-5}$, In Chan Song ${ }^{4,6}$, Seok-ki Kim ${ }^{7,8}$, Daehong Kim ${ }^{8}$, and Jae Sung Lee ${ }^{1-4}$ \\ ${ }^{1}$ Department of Nuclear Medicine, Seoul National University, Seoul, Korea; ${ }^{2}$ Department of Biomedical Sciences, Seoul National \\ University, Seoul, Korea; ${ }^{3}$ Interdisciplinary Program in Radiation Applied Life Science, Seoul National University, Seoul, Korea; \\ ${ }^{4}$ Institute of Radiation Medicine, Medical Research Center, Seoul National University, Seoul, Korea; ${ }^{5}$ Department of Molecular \\ Medicine and Biopharmaceutical Sciences, Graduate School of Convergence Science and Technology, Seoul National University, \\ Suwon, Korea; ${ }^{6}$ Department of Radiology, Seoul National University, Seoul, Korea; ${ }^{7}$ Department of Nuclear Medicine, National \\ Cancer Center, Goyang, Korea; and ${ }^{8}$ Molecular Imaging and Therapy Branch, National Cancer Center, Goyang, Korea
}

Visualization of biologic processes at molecular and cellular levels has revolutionized the understanding and treatment of human diseases. However, no single biomedical imaging modality provides complete information, resulting in the emergence of multimodal approaches. Combining state-of-the-art PET and MRI technologies without loss of system performance and overall image quality can provide opportunities for new scientific and clinical innovations. Here, we present a multiparametric PET/MR imager based on a smallanimal dedicated, high-performance, silicon photomultiplier (SiPM) PET system and a 7-T MR scanner. Methods: A SiPM-based PET insert that has the peak sensitivity of $3.4 \%$ and center volumetric resolution of 1.92/0.53 $\mathrm{mm}^{3}$ (filtered backprojection/ordered-subset expectation maximization) was developed. The SiPM PET insert was placed between the mouse body transceiver coil and gradient coil of a 7-T small-animal MRI scanner for simultaneous PET/MRI. Mutual interference between the MRI and SiPM PET systems was evaluated using various MR pulse sequences. A cylindric corn oil phantom was scanned to assess the effects of the SiPM PET on the MR image acquisition. To assess the influence of MRI on the PET imaging functions, several PET performance indicators including scintillation pulse shape, flood image quality, energy spectrum, counting rate, and phantom image quality were evaluated with and without the application of MR pulse sequences. Simultaneous mouse PET/MRI studies were also performed to demonstrate the potential and usefulness of the multiparametric PET/MRI in preclinical applications. Results: Excellent performance and stability of the PET system were demonstrated, and the PET/MRI combination did not result in significant image quality degradation of either modality. Finally, simultaneous PET/MRI studies in mice demonstrated the feasibility of the developed system for evaluating the biochemical and cellular changes in a brain tumor model and facilitating the development of new multimodal imaging probes. Conclusion: We developed a multiparametric imager with high physical performance and good system stability and

Received Nov. 19, 2015; revision accepted Mar. 11, 2016.

For correspondence or reprints contact either of the following:

Jae Sung Lee, Department of Nuclear Medicine, Seoul National University,

103 Daehak-ro, Jongno-gu, Seoul 110-799, Korea.

E-mail: jaes@snu.ac.kr

Daehong Kim, Molecular Imaging and Therapy Branch, National Cancer

Center, Goyang 410-769, Korea.

E-mail: dkim@ncc.re.kr

Published online Apr. 14, 2016.

COPYRIGHT (C) 2016 by the Society of Nuclear Medicine and Molecular Imaging, Inc. demonstrated its feasibility for small-animal experiments, suggesting its usefulness for investigating in vivo molecular interactions of metabolites, and cross-validation studies of both PET and MRI.

Key Words: PET/MRI; silicon photomultiplier (SiPM); hybrid imaging; multi-parametric imaging; dual-modality imaging probe

J Nucl Med 2016; 57:1309-1315

DOI: 10.2967/jnumed.115.170019

A substantial role of small-animal imaging has been pinpointed in numerous studies in terms of understanding the underlying mechanism of human diseases and elucidating the efficacy of new therapeutic approaches. Among the in vivo small-animal imaging modalities, which are scaled down to dedicated devices from clinical ones, PET is the most-sensitive technique that is readily translatable to the clinic (1). Spatial and temporal distributions of compounds labeled with a positron-emitting radionuclide are noninvasively measured by the PET scanner. Consequently, the PET scanner provides quantitative information about various physiologic and biochemical processes, such as glucose metabolism, gene expression, and drug occupancy (2-5). Additionally, insufficient morphologic information in PET images can be compensated for by combining the PET scanner with morphologic imaging devices, such as CT and MRI.

Despite the great success of PET/CT in both clinical and preclinical applications, a system combining PET and MRI has been demanded because of the advantages of MRI over CT $(6,7)$. Particularly in small-animal imaging studies, the use of MRI reduces radiation exposure to the animal, which needs to be minimized in longitudinal experiments to avoid confounding biologic effects (8). A simultaneously operating PET/MRI system is advantageous in reducing acquisition time and anesthesia dose and in attaining nearperfect spatial and temporal correlation between the information provided by the 2 imaging modalities. In addition, the functional capability of MRI has been dramatically enhanced in the last 2 decades, opening new opportunities for studying pathology and biochemical processes through the combinations of multiparametric MRI and PET.

Early attempts at simultaneous PET/MR image acquisition using long optical fiber bundles did not provide sufficiently good PET 
performance because of the large light loss in the fiber bundles that transfer scintillation light photons generated from the scintillation crystals installed inside the MRI magnet to the photomultiplier tubes located outside the magnet $(9,10)$. To overcome this problem, the magnetically insensitive and compact avalanche photodiode had been suggested as an alternative photosensor for PET detectors and led to the successful development of preclinical and clinical simultaneous PET/MRI systems (11-14). However, the relatively low signal amplification gain and slow time response of avalanche photodiodes compared with photomultiplier tubes have stressed the demand for more advanced semiconductor photosensors in nextgeneration simultaneous PET/MRI systems. In recent years, several groups, including us, have demonstrated the feasibility of smallanimal PET and PET/MR imaging based on silicon photomultipliers (SiPMs); SiPMs are composed of an array of micro-avalanche photodiode cells operating in Geiger mode, which provides a much higher signal amplification gain and faster timing properties than the avalanche photodiode (15-20).

Here, we present an SiPM-based MR-compatible PET imaging system that is combined with a 7-T small-animal dedicated MR scanner. The results of mutual interference evaluation and simultaneously acquired PET/MR images from 3 different mice studies are presented. Collectively, these studies demonstrate that the developed PET/MRI system enables in vivo multiparametric PET/MRI studies with high PET performance and negligible degradation in 7-T MR performance.

\section{MATERIALS AND METHODS}

\section{MR-Compatible SiPM PET System}

The SiPM is a semiconductor photosensor that offers extremely high counting rates and photon number resolution with MR compatibility. We designed the SiPM PET system to be placed between the mouse volume radiofrequency coil and the actively shielded gradient coil of a 7-T small-animal MR scanner (BioSpec 70/20 USR; Bruker Biospin) (Fig. 1; Supplemental Fig. 1 [supplemental materials are available at http://jnm.snmjournals.org]). The radiofrequency and gradient coils have inner/outer diameters of 35/60 and 114/198 mm, respectively. Thus, in designing the SiPM PET insert for the 7-T MR scanner, we focused on minimizing the thickness of the PET system to fit within the narrow

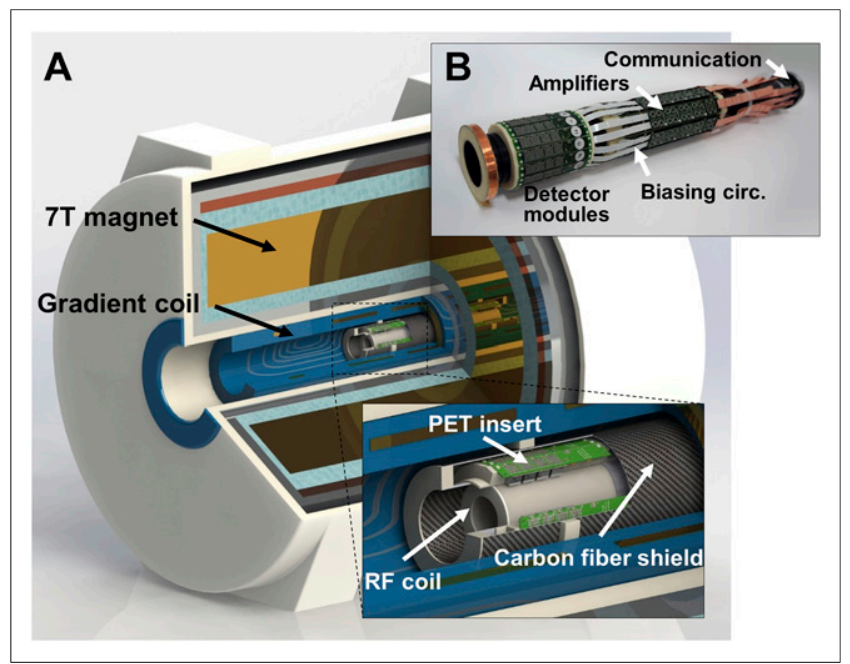

FIGURE 1. (A) Three-dimensional drawing of PET/MR system. (B) Picture of PET insert inside carbon fiber shield shows detector modules, amplifiers, biasing circuits (circ), and communication circuit. space between the coils. Nonmagnetic passive electronic components and connectors were used in the parts placed inside the MR scanning room. The SiPM PET consists of 16 detector modules, and each detector module includes 4 detector blocks. Each detector block is composed of a $9 \times 9$ array of lutetium yttrium orthosilicate (LYSO) crystals $(1.2 \times 1.2 \times$ $10 \mathrm{~mm}^{3}$; SIPAT) and a large-area monolithic $4 \times 4$ SiPM array (S118283344M; Hamamatsu Photonics). Optical grease (BC-630; Oken) was used to optically couple the LYSO crystals and SiPM arrays without inserting any light guides between them. The 16 AC-coupled anode signals of the SiPM array were multiplexed to 4 position signals through a resistive charge division network (21).

To minimize heat generation from the electronics, low-power amplifiers were placed about $15 \mathrm{~cm}$ from the SiPM detectors. We also implemented a real-time gain compensation module based on the temperature monitoring and bias voltage feedback to maintain uniform performance (22). The real-time adjustment of bias voltage yielded stable photopeak positions ( $<0.5 \%$ variation), energy resolutions (average energy resolution was maintained at $14.2 \%$ despite the temperature variation), and prompt counting rates $(<1.2 \%$ variation) over a wide temperature range $\left(10^{\circ} \mathrm{C}-30^{\circ} \mathrm{C}\right)$.

The PET insert was shielded by a $1-\mathrm{mm}$-thick carbon fiber tube (inner/outer diameters, $98 / 100 \mathrm{~mm}$ ), a 3-dimensional (3D) printed front cap coated with a $30-\mu \mathrm{m}$ copper film, and a rear cap made of 1-mm-thick aluminum for electromagnetic shielding. The thicknesses of the shield materials, which were closely located in the PET field of view (FOV), were carefully determined regarding their conductivity (carbon fiber, $5.88 \times 10^{4}$; copper, $5.95 \times 10^{7}$; and aluminum, $3.55 \times 10^{7} \mathrm{~S} / \mathrm{m}$ ) to minimize eddy currents and perform sufficient radiofrequency blocking. The axial FOV and inner diameter of the PET insert were 55 and $60 \mathrm{~mm}$, respectively (Table 1). The centers of the MR magnet and PET axial FOV were aligned for simultaneous PET/MR imaging studies. The temperature, applied bias voltage, and current were continuously monitored during PET/MRI data acquisition.

The PET insert yields superb performance for small-animal imaging (22). The radial/tangential/axial resolutions estimated from reconstructed point source images using ordered-subset expectation maximization were $0.75 / 0.68 / 1.05 \mathrm{~mm}$ at the center of the FOV and 1.46/0.71/ $1.22 \mathrm{~mm}$ at the 14-mm radial off-center. When the filtered backprojection algorithm was used, the radial/tangential/axial resolution ranged from 1.31/ $1.14 / 1.29 \mathrm{~mm}$ at the center of the FOV to $2.18 / 2.11 / 1.58 \mathrm{~mm}$ at the $14-\mathrm{mm}$ radial off-center. The absolute peak sensitivity was $3.36 \%$, with a coincidence time window of $12 \mathrm{~ns}$ and an energy window of $250-750 \mathrm{keV}$. The peak noise equivalent counting rate and scatter fraction were $42.4 \mathrm{kcps}$ at $15.08 \mathrm{MBq}$ and $16.5 \%$ with the same window setting, respectively, for a National Electrical Manufacturers Association mouse-sized phantom.

TABLE 1

Major Characteristics of SiPM PET Insert

\begin{tabular}{lc}
\hline \multicolumn{1}{c}{ Characteristic } & Value \\
\hline Detector face to face $(\mathrm{mm})$ & 64 \\
\hline Scintillator material & LYSO \\
\hline Crystal size $\left(\mathrm{mm}^{3}\right)$ & $1.2 \times 1.2 \times 10.0$ \\
\hline Crystal pitch $(\mathrm{mm})$ & 1.28 \\
\hline No. of crystal rings & 36 \\
\hline No. of crystals/ring & 144 \\
\hline Total no. of crystals & 5,184 \\
\hline Axial FOV $(\mathrm{mm})$ & 55 \\
\hline Insert length $(\mathrm{cm})$ & 82 \\
\hline Insert inner diameter/outer diameter $(\mathrm{mm})$ & $60 / 99$ \\
\hline
\end{tabular}


All PET and PET/MR studies were performed at $20^{\circ} \mathrm{C} \pm 2{ }^{\circ} \mathrm{C}$ with the real-time bias voltage adjustment. PET data were acquired using a custom-made field-programmable gate array (FPGA)-based data acquisition (DAQ) system. The differential position signals (scintillation pulse after passing resistive charge division network) from the amplifiers were digitized by free-running analog-to-digital converters with 12-bit resolution and 125 Msps sampling speed (ADS6425; Texas Instruments). Once a trigger signal was detected, an FPGA (Virtex6; Xilinx) generated a time stamp with a bin size of 2 ns and stored the streams of position signals. The baseline drift was compensated for by subtracting DC level calculated from eight samples preceding the rising edge of the position signal, and then the integral value of each position signal was calculated by integration of signals for $256 \mathrm{~ns}$ (32 samples for $125 \mathrm{Msps}$ ). Single-event information containing DAQ channel number, time stamp, and position signal integral values were transferred to the personal computer via gigabit Ethernet. The list-mode data were saved with the exact detector block position decoded from the DAQ channel number and the position signal integral values in the personal computer. Three-dimensional line-of-response (LOR) histograms of prompt coincidence events were generated from the list-mode data. The energy and timing windows were set to 350 $650 \mathrm{keV}$ and $12 \mathrm{~ns}$, respectively, unless otherwise stated. The LOR histograms were corrected for random coincidence events estimated from single rates. Component-based normalization was performed to compensate for sensitivity variation in LORs, and normalization components were derived by scanning cylinder and annulus phantoms filled with ${ }^{18} \mathrm{~F}-\mathrm{FDG}$ covering the entire FOV. All data were reconstructed using LOR-based, 3D ordered-subset expectation maximization with 3 iterations and 12 subsets and postsmoothed using a 3D Gaussian kernel of $1 \mathrm{~mm}$ in full width at half maximum.

\section{Assessment of Mutual Interference Between PET and MRI}

Influence of PET on MRI. To assess the influence of the PET insert on MR measurement, a uniform corn oil (which has good chemical and thermal stability and appropriate $\mathrm{T} 1, \mathrm{~T} 2$, and proton density values that are within the biological range) phantom with $\mathrm{T} 1$ and $\mathrm{T} 2$ relaxation times of 423 and $130 \mathrm{~ms}$, respectively, was scanned. Main magnetic field (B0) stability, image quality, and temporal stability of image intensity were evaluated following similar procedures used in Wehrl et al. and Hong et al. $(23,24)$. Great care was taken to ensure that the relative positions of the phantom, radiofrequency coil, and main magnet were not changed during the comparative assessment. Prior to obtaining MR scans, the proton resonance spectrum was obtained to verify the effect of the PET insert on the resonant frequency. This procedure was necessary because the off-resonance operation manifests as a reduction in image signal-to-noise ratio (SNR), system sensitivity, and image linearity. SNR and integral uniformity for 4 frequently used MRI sequences in routine animal scans of 2-dimensional (2D) fast spin-echo (FSE), 2D spin-echo (SE), 2D gradientecho (GRE), and 3D spoiled gradient-recalled-echo (SPGR) (Supplemental Table 1) were analyzed using the following equations:

$$
\begin{gathered}
\mathrm{SNR}=0.655 \frac{\mu_{\mathrm{S}}}{\sigma_{\mathrm{N}}} \\
\text { Integral uniformity }=\left[1-\frac{\left(\mathrm{S}_{\max }-\mathrm{S}_{\min }\right)}{\left(\mathrm{S}_{\max }+\mathrm{S}_{\min }\right)}\right] \times 100 \%,
\end{gathered}
$$

where $\mu_{S}$ is the mean signal intensity in a region of interest (ROI) placed in a corn-oil-filled region (ROIs), $\sigma_{\mathrm{N}}$ is the standard deviation of signal intensity derived from an ROI placed in a background region $\left(\mathrm{ROI}_{\mathrm{N}}\right), \mathrm{S}_{\max }$ and $\mathrm{S}_{\min }$ are the maximum and minimum values in ROIs, and the factor 0.655 in the SNR equation was introduced due to Rician distribution of background noise in magnitude images.

B0 inhomogeneity can be caused by PET-induced magnetic susceptibility variations and regional eddy current generations, even when appropriate magnet shimming has been used. Therefore, the B0 distortion was also measured by a phase measurement method. A multiple gradientecho sequence with the following parameters was applied to obtain phantom images in the coronal plane: repetition time (TR), $500 \mathrm{~ms}$; first echo time ( $\left.\mathrm{TE}_{1}\right), 10 \mathrm{~ms}$; second echo time $\left(\mathrm{TE}_{2}\right), 20 \mathrm{~ms}$; third echo time $\left(\mathrm{TE}_{3}\right), 30 \mathrm{~ms}$; FOV, $35 \times 60 \mathrm{~mm}^{2}$; matrix size, $64 \times 128$; slice thickness, $2.0 \mathrm{~mm}$; number of slices, 3; and pixel bandwidth, $257 \mathrm{~Hz} /$ pixel. From the phase map derived from the images obtained with $\mathrm{TE}_{1}\left[\Phi\left(\mathrm{r}, \mathrm{TE}_{1}\right)\right]$ and $\mathrm{TE}_{2}\left[\Phi\left(\mathrm{r}, \mathrm{TE}_{2}\right)\right], \mathrm{B} 0$ maps were generated as follows:

$$
\Delta \mathrm{B}_{0}(r)=\frac{\Phi\left(\mathrm{r}, \mathrm{TE}_{2}\right)-\Phi\left(\mathrm{r}, \mathrm{TE}_{1}\right)}{\gamma\left(\mathrm{TE}_{1}-\mathrm{TE}_{2}\right)},
$$

where $\gamma$ is the gyromagnetic ratio of ${ }^{1} \mathrm{H}$.

Gradient-recalled echo planar imaging (EPI) used in functional MRI studies was evaluated with the following parameters: TR/TE, $2,000 \mathrm{~ms} / 9 \mathrm{~ms}$; flip angle, $30^{\circ}$; FOV, $60 \times 60 \mathrm{~mm}^{2}$; matrix size, $64 \times 80$; slice thickness, $1.5 \mathrm{~mm}$; number of slices, 5; and pixel bandwidth, 67.9 $\mathrm{Hz} /$ pixel. A total of 180 volume scans were acquired over $6 \mathrm{~min}$. The mean signal intensity and percentage temporal drift of the signal (i.e., the standard deviation of signal intensity) on the ROIs were evaluated as a performance indicator in the functional MRI experiments.

Influence of MRI on PET. To assess the influence of MRI on the PET insert installed inside the 7-T magnet, several PET factors including scintillation pulse shape, flood image quality, energy spectrum, count rate, and phantom image quality measured with and without application of radiofrequency sequences were compared. The 2D FSE, 3D SPGR, and EPI sequences with identical parameters used in the assessment of the effects of PET on MRI were used for these experiments, except for the number of excitations (NEX) to make the scan time longer than $5 \mathrm{~min}$ for each acquisition. The scintillation pulses were captured with a 125-Msps analog-to-digital converter used in the DAQ system and examined for baseline distortion and radiofrequency and gradient-induced noise. The flood map quality and energy spectrum, including photopeak fraction and energy resolution, were also evaluated on data obtained with an ${ }^{18} \mathrm{~F}-\mathrm{FDG}$ filled uniform phantom. Blank PET scan data were also acquired to investigate count rate variation based on gradient and radiofrequency pulse applications. The single-event rates triggered by the intrinsic activity of ${ }^{176} \mathrm{Lu}$ of the LYSO crystal were measured in each condition, with a threshold level of approximately $50 \mathrm{keV}$. The uniform and hot-rod phantom filled with ${ }^{18} \mathrm{~F}-\mathrm{FDG}$ was scanned for 30 min to investigate the artifacts in the reconstructed PET images. Care was taken to ensure that the activity used in the simultaneous PET/MR and standalone PET scans was not different $(7.4 \mathrm{MBq} \pm 5 \%)$. The standalone PET scan was obtained outside the MR magnet after the radiofrequency coil was removed.

\section{Animal Experiments}

All animal experiments were approved by the Institutional Animal Care and Use Committee at the National Cancer Center. PET and MR images were fused after spatial registration with a rigid-body transformation between them using AMIDE and FIRE software $(25,26)$. The detailed MR protocols used for animal studies are described in Supplemental Table 2.

\section{Glucose Metabolism Imaging}

To demonstrate the feasibility of the developed PET/MRI system in a mouse neuroimaging study, we examined the metabolic function of the mouse brain. We intravenously injected ${ }^{18} \mathrm{~F}-\mathrm{FDG}$ of $17.2 \mathrm{MBq} / 0.2 \mathrm{~mL}$ via the tail into a BALB/c mouse (female; age, $21 \mathrm{wk}$; weight, $19 \mathrm{~g}$ ) under 
light isoflurane anesthesia. After a 10-min uptake, PET was performed for 60 min simultaneously with high-resolution T2-weighted MRI (TR/TE, 2,500/35 ms; echo train length [ETL], 8; NEX, 16).

\section{Tumor Imaging}

U87MG glioblastoma cells were implanted into the brain of an 8-wk-old female BALB/c nude mouse. Simultaneous imaging study was performed on the mouse at $10 \mathrm{wk}$ of age $(18.4 \mathrm{~g}), 20 \mathrm{~min}$ after intravenous injection of $12.2 \mathrm{MBq}$ of ${ }^{11} \mathrm{C}-\mathrm{MET}$. MR sequences and their parameters used in the study are as follows: FSE (TR/TE, 2,500/35 ms; ETL, 8; NEX, 4), fluid-attenuated inversion recovery (FLAIR; TR/TE, 3,000/10 ms; inversion time, $825 \mathrm{~ms}$; ETL, 2; NEX, 1), and diffusion-weighted imaging (TR/TE, 2,000/27 ms; b values, 0, 45, 350, 1,000, and 2,000 s/mm²). The apparent diffusion coefficient (ADC) map was calculated from the diffusion-weighted images.

\section{Lymph Node (LN) Imaging}

Simultaneous PET/MRI is also a useful tool for developing multimodal imaging probes. An imaging study with ${ }^{64} \mathrm{Cu}$-NOTA-ironoxidemannose $\left({ }^{64} \mathrm{Cu}-\mathrm{NOTA}-\mathrm{IO}-\mathrm{MAN}\right)$, a new dual-modality probe yielding superb T2 contrast in MRI (supplemental data; Supplemental Fig. 2), was performed to demonstrate this (27). We chose the lymphatic system as an in vivo model because of its clinical significance, particularly in oncologic studies in which the invasion status of the LN is crucial information for disease stratification, staging, and management (28).

First, T2-weighted MR images (TR/TE, 2,500/30 ms; ETL, 4; NEX, 4) of the lower abdominal area and legs of an anesthetized BALB/c mouse (female; age, $6 \mathrm{wk}$; weight, $18.5 \mathrm{~g}$ ) including both left and right popliteal LNs in the FOV were acquired. ${ }^{64} \mathrm{Cu}-\mathrm{NOTA}-\mathrm{IO}-\mathrm{MAN}$ $(0.37 \mathrm{MBq} / 10 \mu \mathrm{L})$ was then intradermally injected into the left hind paw of the mouse, carefully maintaining the posture. Simultaneous PET/MRI using the same MRI parameters as the preinjection scan was performed $10 \mathrm{~min}$ and $2 \mathrm{~h}$ after injection. After the image acquisition, the animal was sacrificed using $\mathrm{CO}_{2}$ inhalation, and both left and right popliteal LNs were isolated. The isolated tissue was embedded in paraffin and dissected at $8 \mu \mathrm{m}$. The sections were deparaffinized and then stained with Prussian blue to detect iron oxide particles and counterstained with nuclear fast red. The specimens were dehydrated and mounted on a coverslip, and microscope analysis was performed.

\section{RESULTS}

\section{Influence of PET on MR Performance}

The shift of proton resonance frequency caused by the insertion of the PET system into the MR system was less than $300 \mathrm{~Hz}$ (i.e., $1.0 \mathrm{ppm}$ at $7 \mathrm{~T}$ ) regardless of the PET power on/off condition. There were no perceptible distortions in the MR images acquired with and without the PET insert (Supplemental Fig. 3). Additionally, there were almost no differences in the MR image intensity levels and uniformities with and without PET for various MR imaging sequences used in routine animal studies (Fig. 2A). However, the PET insert induced considerable SNR degradations in MR images, mainly because of the increased variations in background areas, and the SNRs became worse (10\% more degradation) when the power of the PET insert was turned on (Fig. 2A). This result might imply that the quality factor of the radiofrequency coil was degraded by the loading effect of the PET insert and that additional noise was introduced to the radiofrequency coil by the penetrating electromagnetic induction generated from the PET electronics. B0 was slightly distorted $(<0.28 \mathrm{ppm})$ with the PET insert, relative to that without the PET insert $(<0.25 \mathrm{ppm})$, because of the magnetic susceptibility of the PET component, current supplied to the detector modules, and eddy current in the shield materials (Fig. 2B). Nevertheless, both results were well below the maximum field variation allowed in acceptance testing of the Bruker system (1 ppm). In addition, EPI was well preserved without quality degradation and image distortion after the PET system was inserted and its power turned on (Fig. 2C). The relative SD of EPI signal intensity was $0.15 \%$ in all conditions, which was measured over 180 image sets and obtained for $6 \mathrm{~min}$ (Fig. 2C). This result is a solid indication that our PET/MRI system is feasible for multifunctional simultaneous PET/MRI studies because the typical blood oxygen level-dependent signal change level $(5 \%)$ measured by EPI functional MRI studies is much larger than the relative SD $(0.15 \%)$.

\section{Influence of MRI on PET Performance}

The influence of the static 7-T magnetic field and the timevarying radiofrequency waves on the PET system was negligibly small. Neither shape distortion nor baseline drift of PET scintillation pulses due to gradient change and radiofrequency excitation was observed by analyzing scintillation pulses captured with a 125-Msps analog-to-digital converter (Supplemental Fig. 4). No remarkable changes in flood images and energy spectra were observed after radiofrequency sequences were applied (Supplemental Figs. 5 and 6). There was only a slight increase in scattering events caused by radiofrequency coil insertion. When we compared PET images of the uniform phantom acquired with standalone PET and simultaneous PET/MRI, only an additional attenuation in PET images was caused by the radiofrequency coil (Fig. 3A). With the radiofrequency coil, $9 \%$ less prompt counts were collected. Interestingly, the spatial resolution of PET shown in the hot-rod phantom image was better inside the MR scanner (Fig. 3A) as a result of shortened positron range in the strong magnetic field $(29,30)$. Single-event counting rates of PET were 


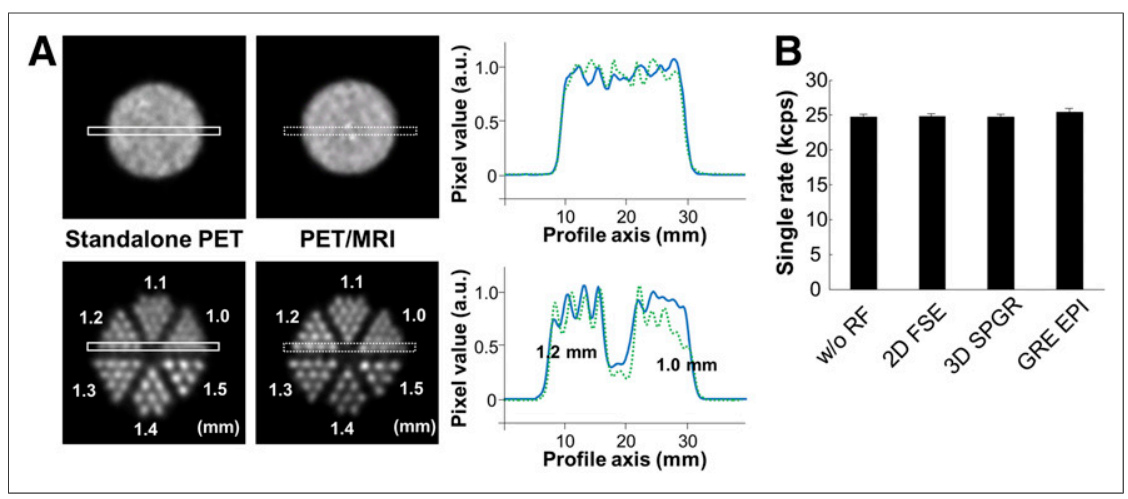

FIGURE 3. (A) Uniform and hot-rod phantom images and profiles across indicated positions acquired under 2 different conditions: after installing PET outside MRI (solid line) and inserting PET into MRI and running 2D FSE sequence (dashed line). Attenuation due to radiofrequency coil was not corrected. (B) PET single-event counting rate measured under 4 different conditions inside MRI: without running any RF pulse and while running 2D FSE, 3D SPGR, and gradient-echo EPI sequences. a.u. = arbitrary units; $\mathrm{RF}=$ radiofrequency.

measured using the intrinsic radioactivity of the LYSO crystal (blank scan), and there was no significant change found when various radiofrequency pulse sequences were applied, except for the gradient-intensive EPI sequence, which yielded a $2 \%$ increment in counting rate (Fig. 3B). The counting rate change would be less perceptible in animal studies in which the actual PET counting rates are much higher than that in a blank scan.

\section{Glucose Metabolism Imaging}

Thanks to the sufficiently long axial FOV of our PET system, the whole mouse brain including the cerebellum and olfactory bulbs could be assessed in a single bed position with uniform spatial resolution (Fig. 4). Moreover, high-resolution 7-T MRI offers excellent anatomic information on the tiny brain structures of the mouse, enabling us to readily examine the ${ }^{18} \mathrm{~F}-\mathrm{FDG}$ distribution in the cerebral cortex and neighboring structures.

\section{Tumor Imaging}

Another primary strength of simultaneous PET/MRI is the diversity of PET radiopharmaceuticals and MRI sequences, offering

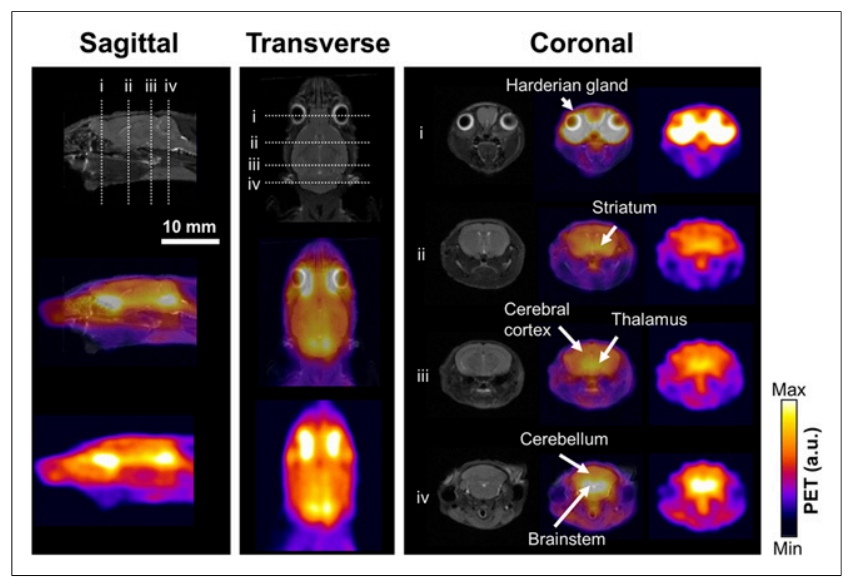

FIGURE 4. Sagittal (left), transverse (center), and coronal (right) images of ${ }^{18} \mathrm{~F}$-FDG PET/MRI. Positions of coronal sections are denoted in sagittal and transverse section by i-iv. High uptake in specific brain regions can be seen including striatum, thalamus, and cerebral cortex. a.u. = arbitrary units; $\max =$ maximum; $\min =$ minimum. perfectly coordinated multiparametric information. The specific uptake of ${ }^{11} \mathrm{C}-\mathrm{MET}$ in U87MG was clearly visualized in PET while T2-weighted and FLAIR MR images showed prominent changes in the tumor (Figs. 5A and 5B; Supplemental Fig. 7). The higher ADC values in U87MG than the surrounding region added to the confidence of tumor detection (Fig. 5C).

\section{LN Imaging}

A left popliteal LN (node draining from the injection site) in the 2-h postinjection MR image resulted in remarkable signal decrease (negative contrast) compared with that in the MR image acquired before injection and was in good agreement with ${ }^{64} \mathrm{Cu}$ signals from PET (Figs. 6A and 6C), providing solid indication of the $\mathrm{LN}$-specific uptake of the tracer. The 10-min postinjection PET also showed clear regional activity in the LN, but the 10-min uptake period was not sufficient to generate MR contrast (Fig. 6B); this is mainly because of the difference in the sensitivities of 2 modalities. The ex vivo examination demonstrated the existence of ferric iron $\left(\mathrm{Fe}^{3+}\right)$ in the left popliteal $\mathrm{LN}$ but not in the right popliteal $\mathrm{LN}$ (Supplemental Fig. 8).

\section{DISCUSSION}

One of the most promising applications of SiPM is simultaneous PET/MR imaging of animal models of human diseases, but there are several technical challenges. This work was motivated by the goals of building a small-animal PET/MRI system with fine spatial resolution, high sensitivity, high temporal stability, and minimal mutual interference and demonstrating the feasibility of this system for various animal experiments.

The developed SiPM PET system achieves submillimeter spatial resolution in the center of the FOV $(0.7 \mathrm{~mm}$ in-plane using iterative image reconstruction of a point source in air) desired for rodent brain imaging and high peak system sensitivity $(>3 \%)$ desired for dynamic PET studies with short-lived radiotracers and short time frames (22). Our new PET system neither significantly degrades the MRI information nor is affected by MRI acquisition. In particular, the minimal

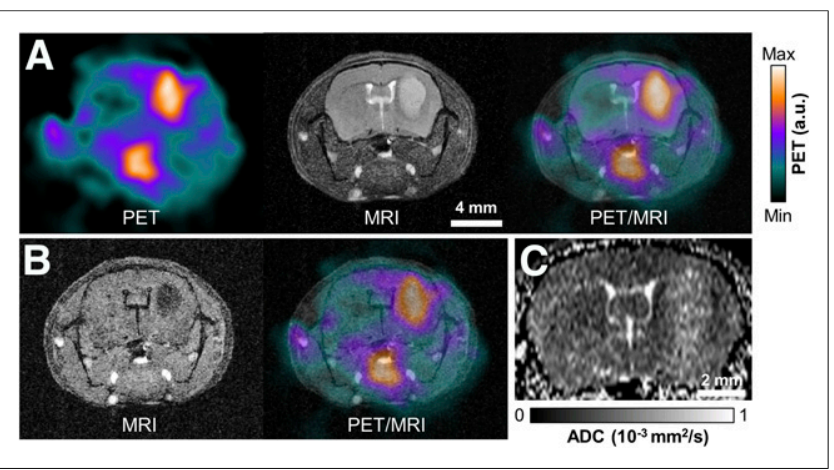

FIGURE 5. (A) Brain tumor images of ${ }^{11} \mathrm{C}-\mathrm{MET}$ PET, T2-weighted FSE MRI, and fused PET/MRI. (B) FLAIR image and fused PET/MRI. (C) Calculated ADC map along $x$-direction. a.u. = arbitrary units; $\max =$ maximum; $\min =$ minimum. 


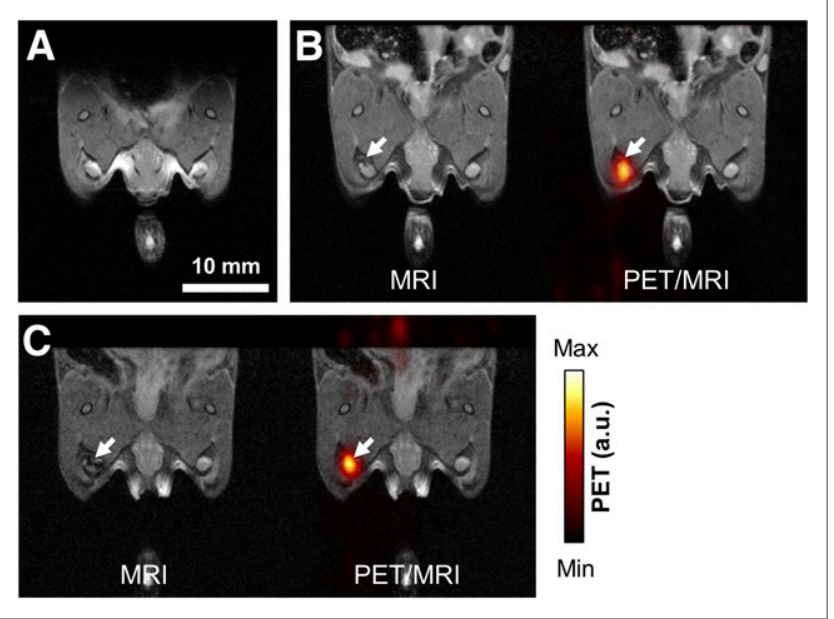

FIGURE 6. (A) T2-weighted MR image of lower abdominal area showing popliteal LN before injection. (B) T2-weighted MR and fused PET/ MR images obtained $10 \mathrm{~min}$ after injection. Arrow indicates draining popliteal LN. (C) T2-weighted MR and fused PET/MR images obtained $120 \mathrm{~min}$ after injection. Signal attenuation is more obvious than 10-min postinjection MR image shown in B. a.u. = arbitrary units; $\max =$ maximum; $\min =$ minimum .

effects of SiPM PET on EPI MR images will allow us to perform simultaneous PET/functional MRI studies, offering a unique methodology for understanding the relationship between hemodynamic and neurochemical processes in the brain $(31,32)$. In addition, the B0 uniformity will enable multiparametric molecular imaging studies with PET tracers and new emerging MR molecular imaging technologies, such as chemical exchange saturation transfer imaging (33).

Our work shows that simultaneous PET/MR imaging with a high-performance SiPM PET and an ultra-high-field small-animal dedicated MR scanner offers synergistic advantages for investigating human disease models in the mouse without significant compromise in the imaging performances of both modalities. The highly specific uptake of ${ }^{11} \mathrm{C}$-MET in the tumor was well correlated with morphologic changes revealed by high-resolution MR images. In addition, parametric MR images of cellular hemodynamics (ADC) incorporated with PET images provide complementary information needed to understand mechanisms underlying the structural alterations in the tumor.

Another important application of simultaneous PET/MRI is the assessment of multimodal imaging probes. Although many interesting multimodal imaging probes have been suggested (34-36), their spatiotemporal characteristics have been investigated using each imaging device separately, making the exact correlation and comparison studies impossible. In contrast, our study with ${ }^{64} \mathrm{Cu}-\mathrm{NOTA}-\mathrm{IO}-\mathrm{MAN}$ showed that high-sensitivity PET images for LN mapping were useful for confirming the accumulation of iron oxide nanoparticles in the same target region. The negative $\mathrm{T} 2$ or $\mathrm{T} 2 *$ contrast enhanced by these MRI agents using just MRI may be hard to distinguish from the inhomogeneity artifacts.

Although the present SiPM-based PET/MRI system showed excellent feasibility in mouse PET/MRI studies, there is room for performance improvements. The traditional multiplexing method used in this study for reducing the number of readout channels (21) somewhat degrades timing and counting rate performances, which can be improved using digital SiPM technology or novel sampling theory $(37,38)$. The improved spatial resolution without the loss of sensitivity will also be possible via the incorporation of depth-ofinteraction measurement technology in PET detector modules (39). In addition, incorporating a total variation minimization algorithm into imaging reconstruction will be useful to improve image quality by reducing gap artifact (40).

\section{CONCLUSION}

We developed a multiparametric imager with high physical performance and good system stability to temperature variation and demonstrated its feasibility for small-animal experiments, suggesting its usefulness for investigating in vivo molecular interactions of metabolites, and cross-validation studies of both PET and MRI. Although we focused on preclinical studies performed inside a small-bore 7-T magnet, the technologies developed in this study can be translated to human PET/MRI scanners with 7-T or higher-field magnets through further technologic developments and appropriate modifications, which will lead the next decade of MRI.

\section{DISCLOSURE}

The costs of publication of this article were defrayed in part by the payment of page charges. Therefore, and solely to indicate this fact, this article is hereby marked "advertisement" in accordance with 18 USC section 1734 . This work was supported in part by grants from the National Research Foundation of Korea (NRF) funded by the Korean Ministry of Science, ICT and Future Planning (2008-2003852 and 2014M3C7034000). The funding source had no involvement in the study design, collection, analysis, or interpretation. No other potential conflict of interest relevant to this article was reported.

\section{ACKNOWLEDGMENTS}

We thank Seung Won Kim and ChangKi Min for assistance with animal preparation and MRI data acquisition, and Yun Sang Park for the design of the 3D printed frame.

\section{REFERENCES}

1. Pomper MG, Lee JS. Small animal imaging in drug development. Curr Pharm Des. 2005;11:3247-3272.

2. Rudin M, Weissleder R. Molecular imaging in drug discovery and development. Nat Rev Drug Discov. 2003;2:123-131.

3. Kang JH, Lee DS, Peang JC, et al. Development of a sodium/iodide symporter (NIS)-transgenic mouse for imaging of cardiomyocyte-specific reporter gene expression. J Nucl Med. 2005;46:479-483.

4. Lee S, Park H. Parametric response mapping of longitudinal PET scans and their use in detecting changes in Alzheimer's diseases. Biomed Eng Lett. 2014;4:73-79.

5. Kim MH, Lee YJ, Kang JH. Stem cell monitoring with a direct or indirect labeling method. Nucl Med Mol Imaging. October 22, 2015 [Epub ahead of print].

6. Pichler BJ, Kolb A, Nagele T, Schlemmer H-P. PET/MRI: paving the way for the next generation of clinical multimodality imaging applications. J Nucl Med. 2010;51:333-336.

7. Drzezga A, Barthel H, Minoshima S, Sabri O. Potential clinical applications of PET/MR imaging in neurodegenerative diseases. J Nucl Med. 2014;55:47S-55S.

8. Hildebrandt IJ, Su H, Weber WA. Anesthesia and other considerations for in vivo imaging of small animals. ILAR J. 2008;49:17-26.

9. Shao Y, Cherry SR, Farahani K, et al. Simultaneous PET and MR imaging. Phys Med Biol. 1997;42:1965-1970.

10. Raylman RR, Majewski S, Lemieux SK, et al. Simultaneous MRI and PET imaging of a rat brain. Phys Med Biol. 2006;51:6371-6379.

11. Judenhofer MS, Wehrl HF, Newport DF, et al. Simultaneous PET-MRI: a new approach for functional and morphological imaging. Nat Med. 2008;14:459-465. 
12. Catana C, Procissi D, Wu Y, et al. Simultaneous in vivo positron emission tomography and magnetic resonance imaging. Proc Natl Acad Sci USA. 2008;105:3705-3710.

13. Schlemmer H-PW, Pichler BJ, Schmand M, et al. Simultaneous MR/PET imaging of the human brain: feasibility study. Radiology. 2008;248:1028-1035.

14. Delso G, Fürst S, Jakoby B, et al. Performance measurements of the Siemens mMR integrated whole-body PET/MR scanner. J Nucl Med. 2011;52:1914-1922.

15. Hong SJ, Song IC, Ito M, et al. An investigation into the use of Geiger-mode solid-state photomultipliers for simultaneous PET and MRI acquisition. IEEE Trans Nucl Sci. 2008;55:882-888.

16. Schaart DR, Van Dam HT, Seifert S, et al. A novel, SiPM-array-based, monolithic scintillator detector for PET. Phys Med Biol. 2009;54:3501-3512.

17. Kwon SI, Lee JS, Yoon HS, et al. Development of small-animal PET prototype using silicon photomultiplier (SiPM): initial results of phantom and animal imaging studies. J Nucl Med. 2011;52:572-579.

18. Roncali E, Cherry SR. Application of silicon photomultipliers to positron emission tomography. Ann Biomed Eng. 2011;39:1358-1377.

19. Yoon HS, Ko GB, Kwon SI, et al. Initial results of simultaneous PET/MRI experiments with an MRI-compatible silicon photomultiplier PET scanner. J Nucl Med. 2012;53:608-614.

20. Yamamoto S, Watabe T, Watabe H, et al. Simultaneous imaging using Si-PMbased PET and MRI for development of an integrated PET/MRI system. Phys Med Biol. 2012;57:N1-N13.

21. Ko GB, Yoon HS, Kwon SI, et al. Development of a front-end analog circuit for multi-channel SiPM readout and performance verification for various PET detector designs. Nucl Instrum Methods Phys Res A. 2013;703:38-44.

22. Ko GB, Kim KY, Yoon HS, et al. Evaluation of a silicon photomultiplier PET insert for simultaneous PET and MR imaging. Med Phys. 2016;43:72-83.

23. Wehrl HF, Judenhofer MS, Thielscher A, Martirosian P, Schick F, Pichler BJ. Assessment of MR compatibility of a PET insert developed for simultaneous multiparametric PET/MR imaging on an animal system operating at 7 T. Magn Reson Med. 2011;65:269-279.

24. Hong SJ, Kang HG, Ko GB, Song IC, Rhee J-T, Lee JS. SiPM-PET with a short optical fiber bundle for simultaneous PET-MR imaging. Phys Med Biol. 2012;57:3869-3883.

25. Loening AM, Gambhir SS. AMIDE: a free software tool for multimodality medical image analysis. Mol Imaging. 2003;2:131-137.

26. Lee JS, Park KS, Lee DS, Lee CW, Chung J-K, Lee MC. Development and applications of a software for functional image registration (FIRE). Comput Methods Programs Biomed. 2005;78:157-164.
27. Yang BY, Moon S-H, Seelam SR, et al. Development of a multimodal imaging probe by encapsulating iron oxide nanoparticles with functionalized amphiphiles for lymph node imaging. Nanomedicine (Lond). 2015;10:1899-1910.

28. Thorek DLJ, Ulmert D, Diop N-FM, et al. Non-invasive mapping of deep-tissue lymph nodes in live animals using a multimodal PET/MRI nanoparticle. Nat Commun. 2014;5:3097.

29. Iida H, Kanno I, Murakami M, et al. A simulation study of a method to reduce positron annihilation spread distributions using a strong magnetic field in positron emission tomography. IEEE Trans Nucl Sci. 1986;33:597-600.

30. Shah NJ, Herzog H, Weirich C, et al. Effects of magnetic fields of up to $9.4 \mathrm{~T}$ on resolution and contrast of PET images as measured with an MR-brainPET. PLoS One. 2014;9:e95250.

31. Wehrl HF, Hossain M, Lankes K, et al. Simultaneous PET-MRI reveals brain function in activated and resting state on metabolic, hemodynamic and multiple temporal scales. Nat Med. 2013;19:1184-1189.

32. Sander CY, Hooker JM, Catana C, et al. Neurovascular coupling to D2/D3 dopamine receptor occupancy using simultaneous PET/functional MRI. Proc Natl Acad Sci USA. 2013;110:11169-11174.

33. Cai K, Haris M, Singh A, et al. Magnetic resonance imaging of glutamate. Nat Med. 2012;18:302-306.

34. Lee H-Y, Li Z, Chen K, et al. PET/MRI dual-modality tumor imaging using arginine-glycine-aspartic (RGD)-conjugated radiolabeled iron oxide nanoparticles. J Nucl Med. 2008;49:1371-1379.

35. Glaus C, Rossin R, Welch $\mathrm{MJ}$, Bao G. In vivo evaluation of ${ }^{64} \mathrm{Cu}$-labeled magnetic nanoparticles as a dual-modality PET/MR imaging agent. Bioconjug Chem. 2010;21:715-722.

36. Yang X, Hong H, Grailer JJ, et al. cRGD-functionalized, DOX-conjugated, and ${ }^{64} \mathrm{Cu}$-labeled superparamagnetic iron oxide nanoparticles for targeted anticancer drug delivery and PET/MR imaging. Biomaterials. 2011;32:41514160 .

37. Haemisch Y, Frach T, Degenhardt C, Thon A. Fully digital arrays of silicon photomultipliers (dSiPM): a scalable alternative to vacuum photomultiplier tubes (PMT). Phys Procedia. 2012;37:1546-1560.

38. van den Berg E, Candès E, Chinn G, et al. Single-photon sampling architecture for solid-state imaging sensors. Proc Natl Acad Sci USA. 2013;110:E2752E2761.

39. Ito M, Hong SJ, Lee JS. Positron emission tomography (PET) detectors with depth-of-interaction (DOI) capability. Biomed Eng Lett. 2011;1:70-81.

40. Son J, Kim SM, Lee JS. A strategy to reduce blocky pattern and contrast loss in emission tomography reconstruction with reduced angular sampling and total variation minimization. Biomed Eng Lett. 2014;4:362-369. 